\title{
Reconstruction of Defect after Treatment of Bisphosphonate-related Osteonecrois of the Jaw with Staged lliac Bone Graft
}

\author{
Kyo-Jin Ahn, Young-Kyun Kim, Pil-Young Yun \\ Department of Oral and Maxillofacial Surgery, Section of Dentistry, Seoul National University Bundang Hospital
}

\begin{abstract}
Bisphosphonate is used widely for osteoporosis treatment, but a rising concern is the risk of osteonecrosis after long-term bisphosphonate use. Such cases are increasing, suggesting a need for research to prevent and treat bisphosphonate-related osteonecrosis of jaws. A 63-year-old female took bisphosphonate (Fosamax ${ }^{\circledR}$ ) for four years for treatment of osteoporosis and stopped medication two months ago because of unhealed wound. She was treated with marginal mandibulectomy maintaining the inferior border, and a metal plate was placed to prevent mandible fracture. Four months after the mandibulectomy, mandible reconstruction surgery using iliac bone and allograft was done. Six months after reconstruction, implant placement and treatment with an overdenture was done without complications. This study presents a case with a successful result.
\end{abstract}

Key words: Bisphosphonate-related osteonecrosis of jaws, Osteonecrosis, Implant, Overdenture

\section{Introduction}

Bisphosphonate is used to prevent pathological fracture resulting from osteoporosis or the bone metastasis of malignant tumors. Bisphosphonate-related osteonecrosis of jaws (BRONJ) was first reported by Marx[1] in 2003, and cases are increasing across the world, including Korea. It raises concern as a new disease in the oral and maxillofacial area, and research is active. The essence of BRONJ has not been accurately identified except that it is rare. Once BRONJ occurs, however, it is difficult to treat, making prevention the preferred approach for high-risk patients.

The American Association of Oral and Maxillofacial
Surgeons presented guidelines in 2006 and 2009 regarding BRONJ[2,3] and in Korea, the Korean Endocrine Society, Korean Society for Bone and Mineral Research, The Korean Society of Osteoporosis and The Korean Association of Oral and Maxillofacial Surgeons jointly presented guidelines in 2009 on BRONJ risks, prevention and therapies[4]. According to these guidelines, a case may be defined as BRONJ if all the following conditions are satisfied.

- When bone is exposed on the jaw region and the symptoms continue for more than eight weeks without healing despite appropriate treatment.

- When the patient took bisphosphonate in the past or is currently taking it.

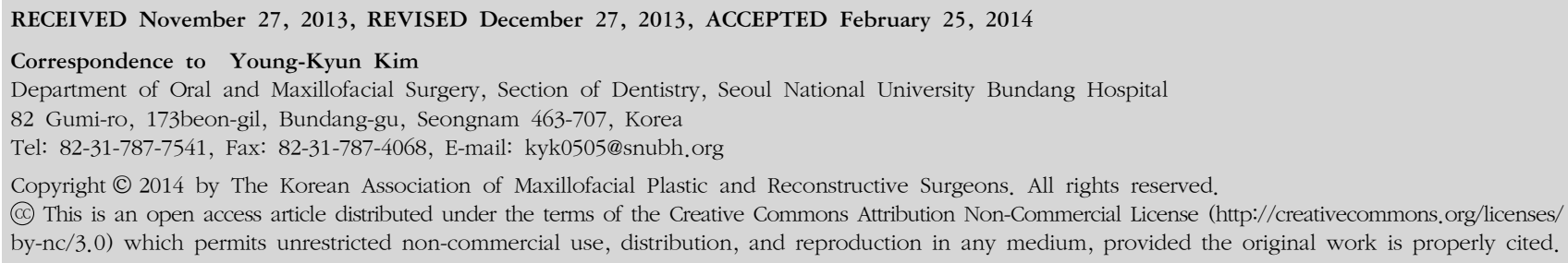


- When the patient did not receive radiation treatment on the jaw region in the past.

The number of patients meeting these criteria in clinical trials is increasing and it is believed that we need more research on development risks, prevention and treatment methods. With BRONJ patients or patients receiving treatment with bisphosphonate through oral preparations or injections, implant-based treatment is being researched. However, there is no established theory requiring additional research. Conservative treatment is recommended but surgical treatment such as bone resection is considered when there is repeated failure of conservative treatment or extensive osteonecrosis. In addition, for implant placement, it is necessary to reconstruct the bone defect. Various methods are used for reconstruction of bone defect. Yoshimura et al.[5] reported a segmental mandibular resection, and immediate reconstruction was done with iliac bone, followed by implant placement seven months after reconstruction. They found that the implant was functioning successfully for ten years. Mücke et al.[6] achieved a reliable reconstruction result with microvascular osteocutaneous fibula and iliac crest flap after radical mandibular resection. We performed marginal mandibulectomy on a 63-year-old female patient suffering from BRONJ, executed mandible reconstruction using autogenous iliac bone and allogenous bone and successfully performed implant placement. The patient was further treated with an implant-supported overdenture that is successfully functioning. Since this significantly improved the quality of life by enhancing patient's functional and esthetic satisfaction, we would like to report the case.

\section{Case Report}

In September 2009, a 63-year-old female patient presented for treatment of a mandibular anterior swelling and delayed wound healing. The patient's right mandibular canine and primary premolar were extracted four months earlier at a dental clinic. However, the wound had not healed, with continued exposure of the extraction socket, gingival swelling, spontaneous pain, and sensory paralysis. The patient said that she started taking bisphosphonate (Fosamax $^{\circledR}$; Merk, Whitehouse Station, NJ, USA) four years ago to treat osteoporosis but stopped two months ago. In the computed tomography image, an osteolytic lesion was observed on the right mandibular body and the symphysis, and the bone scan showed increased hot uptake in the same region. We provisionally diagnosed it as BRONJ and performed marginal mandibulectomy on \#33 45 region preserving mandibular inferior border on September 30, 2009. In addition, reconstruction metal plates were used to prevent bone fracture. Two months after the surgery, all related symptoms were resolved. In January 2010, we executed mandibular reconstruction using autogenous iliac bone and allogeneic bone graft (DBX putty; Synthes, Paoli, PA, USA). The first surgery was done with two implant placements in July 2010, and the second surgery was performed in November 2010. Finally, the overdenture was delivered in December 2010. For the 23 months since, the implants are functioning successfully without complications (Fig. 1 5 ).
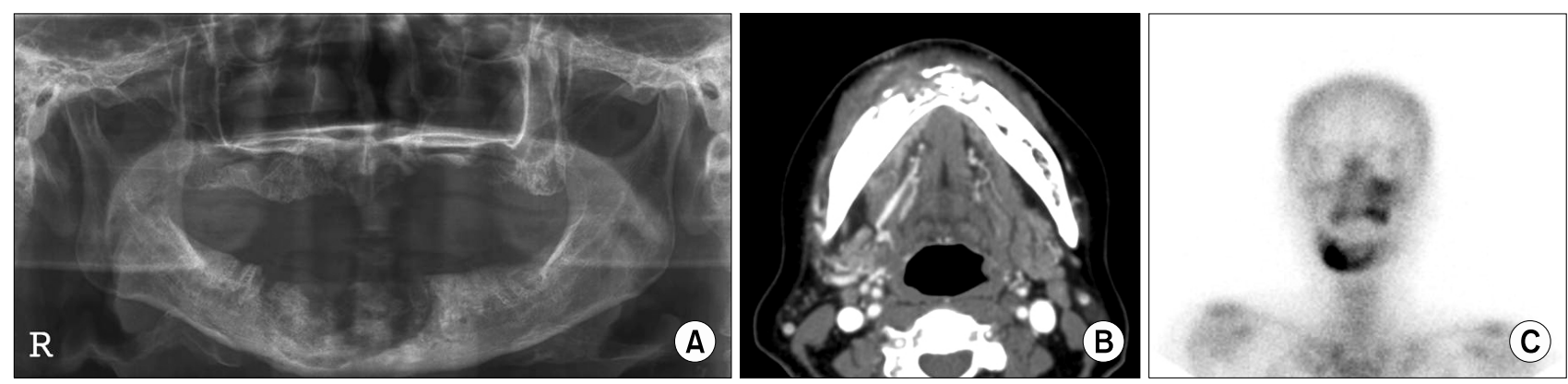

Fig. 1. (A) Initial panoramic view showing an extensive bisphosphonate-related osteonecrosis of jaws of \#33 45 area. The bone appears abnormal and mottled. (B) Initial axial computed tomography scan showing abnormal necrotic bone and intraosseous sequestra. (C) Initial bone scan showing increased hot spots in the mandible corresponding with the clinical and radiological situation. 

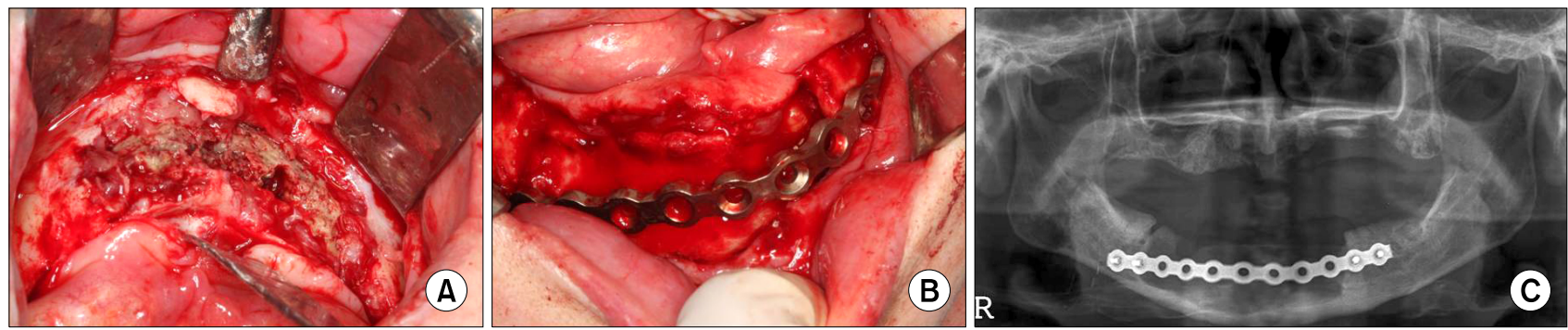

Fig. 2. (A) Intraoral view showing extensive osteonecrosis on $\# 33 \sim 45$ area. (B) Intraoral view showing reconstruction plate fixation after marginal mandibulectomy without bone graft. (C) Postoperative panoramic view showing partially resected mandible and reconstruction plate remaining inferior border.
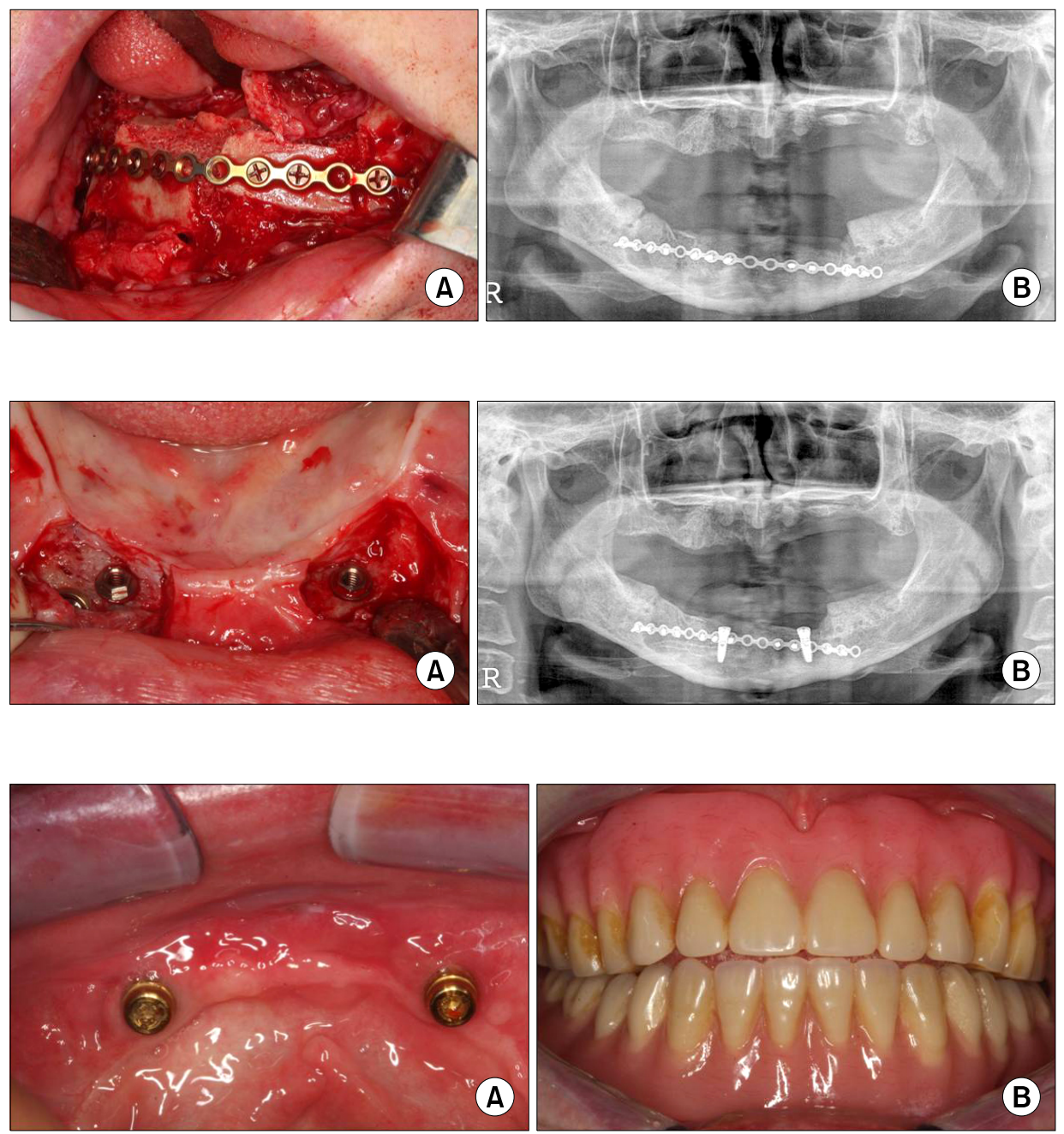

Fig. 3. (A) Four months after mandibular resection, three pieces of iliac block bone were adapted on the defect site and fixated with miniplate. Allogenous bone (DBX putty) was covered between block bones and miniplate. (B) Postoperative panoramic view showing adapted iliac bone and miniplate fixation.

Fig. 4. (A) Six months after bone graft, implant first surgery was performed on \#33 and 43 area. (B) Panoramic view after implant placement showing two implants and well maintaining grafted bone.

Fig. 5. (A) Four months after implant placement, second surgery was performed and locators were placed. (B) Intraoral view showing complete denture on maxilla and overdenture on mandible. They have functioned successfully without any complications for 23 months.

\section{Discussion}

In this case, BRONJ was diagnosed in a patient who took bisphosphonate for four years. Most researchers agree that the risk of BRONJ is lower with oral bisphosphonate than when injection bisphosphonate is used. However, there is a significant difference of opinion regarding the incidence rate of necrosis of the jaw from oral bisphosphonate. The reported incidence rate of BRONJ resulting from Pamidronate (Aredia; Novartis, East Hanover, NJ, USA) and Zoledronate (Zometa; Novartis), the representative bisphosphonate injections, is $6.7 \%$, extremely high[7]. Most research reports that the risk from bisphosphonate injections is high, but Mattheos et al.[8] re- 
cently presented research results stating that if only zoledronic acid is administered to treat osteoporosis by intravenous injection, it is not an absolute contraindication for implant placement. The BRONJ incidence rate when oral bisphosphonate was administered was $0 \%$ to $0.04 \%$, lower than that of injection bisphosphonate, and even the American Dental Association recommended in 2008 it is not necessary to choose particularly conservative treatment for overall dental treatment including implant placement in case of oral bisphosphonate intake[9-12]. However, Sedghizadeh et al.[13] reported that there was jaw necrosis in approximately $4 \%$ of the patients who took oral bisphosphonate. This case also shows jaw necrosis developing in a patient taking oral bisphosphonate with history of extraction. Many other researchers reported that large numbers of BRONJ patients had extraction history, concluding that extraction raises the risk of BRONJ[13-15]. Thus, even if oral medicine is used, the patient and physician should be aware of the risk of osteonecrosis or failure of implant prior to implant placement when bisphosphonate is administered for more than three years[4]. Furthermore, the American Association of Oral and Maxillofacial Surgeons and a joint position statement of Korean domestic societies recommended that bisphosphonate be stopped for three months prior surgical procedures if bisphosphonate was administered for more than three years, or if steroid was administered simultaneously when if intake is less than three years[3,4]. However, there is no evidence that this will improve surgical outcomes.

BRONJ treatment has long been controversial. While conservative treatment is recommended[10], recent studies say that early surgical treatment might be more effective[16,17]. According to the stage and therapeutic strategies presented in 2009 by the American Association of Oral and Maxillofacial Surgeons, BRONJ is classified into four stages along with therapeutic strategies. The 0 stage is that patients complain about non-specific symptoms without any necrotized bone, undergo symptomatic treatment, and antibiotics if needed. The first stage is when there is an exposure of the necrotized bone but no symptoms and no evidence of infection. In this stage, oral irrigation is executed by sterilized oral cleaner (chlorhexidine) and no surgery is needed. The second stage is when there is evidence of pain and infection along with the exposure of the necrotized bone. Oral antibiotics are necessary, oral irrigation is carried out using sterilized oral cleaner, and only the surface layer has to be removed from the necrotized tissue. The third stage occurs when the exposed region of the necrotized bone crosses over the alveolar bone, with accompanying pathological fracture, extra-oral fistula, oro-nasal fistula or sinus tract is formed, or osteolysis has progressed below the mandibular. In this stage, it is necessary to administer general antibiotics and remove the necrotized bone along with oral irrigation[3]. Of these stages, surgical procedure can be considered in the second and third stage. This case is classified as third stage, and it was possible successfully heal bone through early surgical treatment. After bone healing, implants may be placed and the overdenture can function successfully using these implants. We performed staged reconstruction in this case with reconstruction done four months after mandibular resection. In staged reconstruction, the risk of secondary infection is less, there can be sufficient healthy tissue to cover the graft area, and the patient can be in optimal condition for additional surgery. Moreover, surgeons can reassess the esthetic result of reconstructive plan. On the other hand, there are disadvantages of re-approaching the surgical site, with scarring and loss of space to accommodate a reconstruction[18,19]. In this case, the overdenture was delivered after placement of two implants on the mandible. If a conventional denture was used without any implant placement, the denture could not be sufficiently retained. Because the denture continuously stimulates the inferior mucous membrane, there could be damage and ulceration of soft tissue, followed by possible exposure of the bone with a risk of secondary infection. In addition, continuous atrophy may occur on the edentulous region. However, in this case, since implants were placed and the implant-supported overdenture functioned successfully, it was possible to overcome all such problems.

\section{References}

1. Marx RE. Pamidronate (Aredia) and zoledronate (Zometa) induced avascular necrosis of the jaws: a growing epidemic. J Oral Maxillofac Surg 2003;61:1115-7.

2. American Association of Oral and Maxillofacial Surgeons: 2006 Position Paper on Bisphosphonate-Related Osteonecrosis of the Jaws [Internet]. Rosemont (IL): American Association of Oral 
and Maxillofacial Surgeons [cited 2006 Sep 25]. Available from: http://www.aaoms.org/docs/position_papers/osteonecrosis.pdf.

3. Task Force on Bisphosphonate-Related Osteonecrosis of the Jaws: American Association of Oral and Maxillofacial Surgeons Position Paper on Bisphosphonate-Related Osteonecrosis of the Jaw-2009 Update. Approved by the Board of Trustees [Internet]. Rosemont (IL): American Association of Oral and Maxillofacial Surgeons [cited 2009 Jan 15]. Available from: http://www.aaoms.org/docs/position_papers/bronj_update.pdf.

4. Bisphosphonate Related Osteonecrosis of the JAW (BRONJ), Position Statement of Korea [Internet]. Seoul: Korean Endocrine Society [cited 2009 Jun 25]. Available from: http://www.kaoms. org/file/BRONJ_Korea_Position_Statement.pdf.

5. Yoshimura H, Ohba S, Nakamura M, Sano K. Mandibular reconstruction using iliac bone and great auricular nerve grafts and oral rehabilitation using osseointegrated implants in a patient with a large ossifying fibroma: a 10-year follow-up study. J Oral Maxillofac Surg 2013;71:2176-88.

6. Mücke T, Haarmann S, Wolff KD, Hölzle F. Bisphosphonate related osteonecrosis of the jaws treated by surgical resection and immediate osseous microvascular reconstruction. J Craniomaxillofac Surg 2009;37:291-7.

7. Bamias A, Kastritis E, Bamia C, et al. Osteonecrosis of the jaw in cancer after treatment with bisphosphonates: incidence and risk factors. J Clin Oncol 2005;23:8580-7.

8. Mattheos N, Caldwell P, Petcu EB, Ivanovski S, Reher P. Dental implant placement with bone augmentation in a patient who received intravenous bisphosphonate treatment for osteoporosis. J Can Dent Assoc 2013;79:d2.

9. Grbic JT, Landesberg R, Lin SQ, et al; Health Outcomes and Reduced Incidence with Zoledronic Acid Once Yearly Pivotal Fracture Trial Research Group. Incidence of osteonecrosis of the jaw in women with postmenopausal osteoporosis in the health outcomes and reduced incidence with zoledronic acid once yearly pivotal fracture trial. J Am Dent Assoc 2008;139: 32-40.

10. Boonyapakorn T, Schirmer I, Reichart PA, Sturm I, Massenkeil G. Bisphosphonate-induced osteonecrosis of the jaws: prospective study of 80 patients with multiple myeloma and other malignancies. Oral Oncol 2008;44:857-69.

11. Cartsos VM, Zhu S, Zavras AI. Bisphosphonate use and the risk of adverse jaw outcomes: a medical claims study of 714,217 people. J Am Dent Assoc 2008;139:23-30.

12. Edwards BJ, Hellstein JW, Jacobsen PL, Kaltman S, Mariotti A, Migliorati CA; American Dental Association Council on Scientific Affairs Expert Panel on Bisphosphonate-Associated Osteonecrosis of the Jaw. Updated recommendations for managing the care of patients receiving oral bisphosphonate therapy: an advisory statement from the American Dental Association Council on Scientific Affairs. J Am Dent Assoc 2008;139:1674-7.

13. Sedghizadeh PP, Stanley K, Caligiuri M, Hofkes S, Lowry B, Shuler CF. Oral bisphosphonate use and the prevalence of osteonecrosis of the jaw: an institutional inquiry. J Am Dent Assoc 2009;140:61-6.

14. Hansen T, Kunkel M, Weber A, James Kirkpatrick C. Osteonecrosis of the jaws in patients treated with bisphosphonates - histomorphologic analysis in comparison with infected osteoradionecrosis. J Oral Pathol Med 2006;35:155-60.

15. Walter C, Grötz KA, Kunkel M, Al-Nawas B. Prevalence of bisphosphonate associated osteonecrosis of the jaw within the field of osteonecrosis. Support Care Cancer 2007;15:197-202.

16. Stockmann P, Vairaktaris E, Wehrhan F, et al. Osteotomy and primary wound closure in bisphosphonate-associated osteonecrosis of the jaw: a prospective clinical study with 12 months follow-up. Support Care Cancer 2010;18:449-60.

17. Pautke C, Bauer F, Otto S, et al. Fluorescence-guided bone resection in bisphosphonate-related osteonecrosis of the jaws: first clinical results of a prospective pilot study. J Oral Maxillofac Surg 2011;69:84-91.

18. Marx RE. Reconstruction of defects caused by bisphosphonateinduced osteonecrosis of the jaws. J Oral Maxillofac Surg 2009; 67:S107-19.

19. Ferretti C, Rikhotso E, Muthray E, Reyneke J. Interim reconstruction and space maintenance of mandibular continuity defects preceding definitive osseous reconstruction. Br J Oral Maxillofac Surg 2013;51:319-25. 